\title{
Cardiotoxicity-current recommendations of prevention and treatment
}

\author{
Jutta Bergler-Klein (iD)
}

Received: 21 August 2021 / Accepted: 27 September 2021 / Published online: 4 November 2021

(C) The Author(s) 2021

Summary Tremendous advances in modern oncology therapies enable an increasing life expectancy of many cancer entities. Short or long-term cardiovascular side effects, however, gain importance. The current review focuses on recent recommendations for strategies of preventing and treating cardiotoxicity. A personalized assessment of the baseline risk of cardiotoxicity is recommended in all patients, without delaying the initiation of the cancer therapy. A baseline ECG, biomarkers (NTproBNP, troponin), blood pressure and echocardiography should be obtained in all patients scheduled for potentially cardiotoxic treatments. Cardiac risk factors, e.g., coronary disease, hypertension, elevated lipids, should be promptly treated and optimized. Increased surveillance with more frequent cardiac imaging and sequential biomarker assessment during the cycles is recommended in high-risk cardiac patients. New imaging methods in echocardiography such as speckle tracking global longitudinal strain reflecting early myocardial ventricular deterioration are proposed in recent recommendations. Signs of cardiotoxicity should induce early treatment by, e.g., ACE-inhibitors, beta-blockers and/or other heart failure therapies. Immune therapies, e.g., checkpointinhibitors can induce cardiac events such as arrhythmias, acute coronary syndrome with plaque rupture, or myocarditis, even in negative magnetic resonance imaging or normal echocardiography findings. Troponin, BNP and ECG may help to identify these potentially deleterious side effects. Furthermore, there is a bidirectional influence of heart disease and cancer,

A.o. Univ. Prof. J. Bergler-Klein, MD, FESC, FEACVI, FHFA $(\bowtie)$

Department of Cardiology, Medical University of Vienna, Waehringer Guertel 18-20, 1090 Vienna, Austria jutta.bergler-klein@meduniwien.ac.at e.g., by common inflammatory pathways. Pre-existent heart disease leads to worse prognosis in cancer, necessitating close follow-up and cardiac treatment during cancer therapy. On the other hand, cardiovascular mortality is increased after cancer survival and periodic cardiac follow-up is recommended longterm especially after chemotherapy and-or radiation.

Keywords Cardio-oncology · Cardiac side effects chemotherapy · Cardiac surveillance in cancer therapy · Biomarkers · Echocardiography $\cdot$ Speckle tracking strain

\section{Introduction}

Significant advances in modern oncology therapies have led to increasing life expectancy of many cancer entities [1]. Therefore, short- and long-term cardiac side effects of oncology treatments progressively gain importance. Modern imaging methods and biomarkers can help to identify and predict cardiotoxicity in patients undergoing cancer therapies [2]. This review focuses on recent recommendations for strategies of preventing and treating cardiotoxicity.

\section{Baseline individual risk assessment}

In the recent position statement of the Heart Failure Association (HFA) of the European Society of Cardiology (ESC), a personalized approach evaluating the baseline risk of cardiotoxicity is recommended [3]. Patients scheduled to receive potentially cardiotoxic cancer therapies are stratified into three categories (low, medium, or high risk), depending on therapyand patient-related factors [3]. Therapy-related factors include type and dose of anticancer agents, as well as previous cancer therapies (e.g., anthracyclines, radiation), whereas patient-related factors consist of 
age, pre-existent cardiovascular risk factors such as coronary disease, hypertension, heart failure, atrial fibrillation, valve disease, or other comorbidities such as diabetes [3]. Specific proformas may be applied to quantify the overall risk in a total point score [3].

A reduced or even low-normal/borderline left ventricular ejection fraction (LVEF) of $50-54 \%$ in echocardiography before start of cancer therapy categorizes the patient at increased risk of cardiotoxicity [2]. It is therefore essential to assess the myocardial function even before cancer treatment in order to define a starting point for eventual deterioration during and/or after chemotherapy [2, 4]. Similarly, baseline elevation of the serum biomarkers troponin and/or B-type natriuretic peptide (BNP or NT-proBNP) point to an increased cardiotoxicity risk and further cardiac assessment may be needed.

It is highly endorsed that the baseline cardiovascular examinations should not delay the start of the cancer treatment [5]. If a high cardiac risk is identified, this should prompt initiation or optimization of cardiac treatment of many modifiable cardiac risk factors such as hyperlipidemia, hypertension, heart failure or coronary disease. If appropriate, possibly less cardiotoxic oncology therapy regimes may be considered, e.g., liposomal anthracyclines, or the addition of dexrazoxane. A multidisciplinary approach of cardiooncology is emphasized in order to maximize possible benefit of cancer treatment in the individual patient despite any concomitant risk [5].

\section{Cardiovascular surveillance, echocardiography, and biomarkers}

Higher surveillance with more frequent cardiac imaging and biomarker assessment of serum troponin and BNP during the cycles is recommended in high-risk patients. The recent joint position paper on the role of cardiovascular imaging in cardiotoxic cancer treatments of the joint HFA/ESC and EACVI (European As- sociation of Cardiovascular Imaging) depicts guidance especially for the timelines of echocardiography [4]. Local resources and availability of imaging may vary however, and costs may also limit current application.

A summary of how often echocardiography including left ventricular ejection fraction (LVEF) and speckle tracking global longitudinal strain (GLS) should be performed during specific therapies is shown in Table 1.

Different definitions of cardiotoxicity have been proposed. Recently, the ESC and EACVI, as well as ASE (European and American Society of Echocardiography), have defined cancer therapeutics-related cardiac dysfunction (CTRCD) as a decline in LVEF by $>10 \%$ points below the LVEF cut-off $50 \%$ (EACVI/ASE: $53 \%$ ). However, a normal LVEF as measured by the echocardiography biplane Simpson method does not always exclude underlying myocardial dysfunction. An inter- and intraobserver variability of up to $10 \%$ of LVEF measurements has been reported which would confuse cardiotoxicity interpretation.

Early subclinical myocardial damage may be identified by the recently established method of speckletracking echocardiography (global longitudinal strain, GLS) which also relates to elevated BNP. A reduction of GLS by $15 \%$ from baseline has been defined as pointing to the risk of developing consecutive LVEF reduction with overt cardiotoxicity. An impairment of GLS should prompt initiation of preventive cardiac heart failure therapy such as angiotensin converting enzyme inhibitors (ACEI) or angiotensin receptor blockers (ARB), and/or beta-blockers.

Similarly, a rise in BNP or troponin should lead to intensified cardiac treatment (e.g., ACE inhibitors, beta-blockers) and more frequent imaging, as suggested in the recent position paper on biomarkers [6]. Troponin reflects myocardial cell necrosis, e.g., in anthracyclines, or may be elevated in arrhythmias such as tachycardia atrial fibrillation, or due to renal insufficiency. A steep rise during immune checkpoint

\begin{tabular}{|c|c|c|c|c|c|}
\hline & \multirow[t]{2}{*}{ Baseline } & \multirow[t]{2}{*}{ During therapy } & \multicolumn{3}{|c|}{ After completion } \\
\hline & & & Comment & & Comment \\
\hline $\begin{array}{l}\text { Trastuzumab (in early } \\
\text { invasive disease) }\end{array}$ & Yes & Every 4 cycles & $\begin{array}{l}\text { Every } 2 \text { cycles in high risk, } \\
\text { every } 3 \text { cycles in medium risk }\end{array}$ & 6 months after final cycle & $\begin{array}{l}3 \text { and } 12 \text { months after } \\
\text { final cycle in high risk }\end{array}$ \\
\hline \multirow{2}{*}{$\begin{array}{l}\text { Trastuzumab in } \\
\text { metastatic disease } \\
\text { (long-term therapy) }\end{array}$} & \multirow[t]{2}{*}{ Yes } & \multirow[t]{2}{*}{ Every 4 cycles } & Every 6 months when stable & \multirow{2}{*}{$\begin{array}{l}\text { Not indicated unless symp- } \\
\text { tomatic }\end{array}$} & \multirow[t]{2}{*}{ - } \\
\hline & & & $\begin{array}{l}\text { More frequent in medium to } \\
\text { high risk: every } 2-3 \text { cycles }\end{array}$ & & \\
\hline Anthracyclines & Yes & $\begin{array}{l}\text { After completing cumulative } \\
\text { does of } 240 \mathrm{mg} / \mathrm{m}^{2} \text { doxorubicin }\end{array}$ & $\begin{array}{l}\text { Every } 2 \text { cycles in medium to } \\
\text { high risk }\end{array}$ & $\begin{array}{l}\text { 6-12 months after final } \\
\text { cycle (depending on risk) }\end{array}$ & $\begin{array}{l}\text { Reassess after } 5 \text { years } \\
\text { (earlier in high risk) }\end{array}$ \\
\hline $\begin{array}{l}\text { VEGF and Bcr-Abl } \\
\text { TKls }\end{array}$ & $\begin{array}{l}\text { In high-risk } \\
\text { patients }\end{array}$ & $\begin{array}{l}\text { Every } 4 \text { months during the first } \\
\text { year }\end{array}$ & $\begin{array}{l}\text { Every 6-12 months, when long- } \\
\text { term therapy is necessary }\end{array}$ & No clear recommendations & - \\
\hline Proteasome inhibitors & Yes & Every 6 months & Look for signs of amyloidosis & No clear recommendations & - \\
\hline $\begin{array}{l}\text { Immune checkpoint } \\
\text { inhibitors }\end{array}$ & $\begin{array}{l}\text { Yes (depending } \\
\text { on baseline risk) }\end{array}$ & $\begin{array}{l}\text { Immediately when cardiac } \\
\text { symptoms occur. Every } \\
6-12 \text { months in long-term } \\
\text { in high risk }\end{array}$ & CME if myocarditis suspected & No clear recommendations & Consider in high risk \\
\hline
\end{tabular}


Table 2 Confounders influencing serum troponin increase during cancer therapy

Coronary syndrome, plaque rupture

Vasospasm

Tako-Tsubo syndrome

Myocardial necrosis in anthracycline therapy, trastuzumab, radiation

Renal failure

Pericarditis

Myocarditis

Anemia

Sepsis

Blood pressure: hypertensive crisis, hypotension

Arrhythmias, e.g., atrial fibrillation, tachycardia

Hypertrophic cardiomyopathy

Amyloidosis

Pulmonary thromboembolism

Direct metastatic myocardial infiltration

inhibitor therapy may identify immune myocarditis. Troponin increase may also point to ischemia with underlying coronary disease, hypertension or vasospasm, e.g., in fluorouracil (5-FU), capecitabine, or tyrosine kinase inhibitors, or plaques rupture in acute coronary syndromes necessitating coronary angiography and percutaneous coronary intervention (PCI; Table 2). BNP may be influenced by left ventricular volume status and pressure increase (Table 3 ).

Not only heart failure-cardiotoxicity also presents as arrhythmias, atrial fibrillation, e.g., in ibrutinib, or ventricular tachycardias, QT prolongation, hypertension, coronary syndromes and vascular disorders [7].

\section{Bidirectional influence of heart and cancer}

Cross talk and common pathways between tumor and the heart may induce release of biomarkers even before cancer therapy is started [8]. Hypoxia has been shown to trigger cancer growth in a mice model of myocardial ischemia by secretion of circulating factors inducing intestinal tumors, and a higher cumulative incidence of cancer in patients with heart failure 30 days after myocardial infarction was observed [9, 10]. Cardiovascular disease may promote cancer occurrence and progression. Inflammatory pathways, clonal hematopoiesis, hypoxia, as well as circulating microRNAs have been implicated in both atherosclerosis and cancer development, entitled as "reverse cardio-oncology" [11]. These common pathways emphasize the importance of optimal cardiac and heart failure therapy in order to prevent tumor incidence and/or progression.

\section{Case}

A 64-year-old woman with bilateral hereditary breast cancer (HOBC; right breast: invasive lobular carcinoma with ductal carcinoma in situ [DCIS], estro-
Table 3 Confounders influencing serum NT-pro-BNP and increase during cancer therapy

Volume changes, intravenous fluid load, e.g., of chemotherapy

Renal failure

Anemia

Sepsis

Age, weight, gender

Blood pressure, hypertension

Arrhythmias, e.g., atrial fibrillation, tachycardia

Pulmonary thromboembolism

Coronary ischemia

Stiff heart, diastolic dysfunction, e.g., due to radiation

Myocarditis

Hypertrophic cardiomyopathy

Amyloidosis

Carcinoid valve disease

gen receptor [ER] positive, progesterone receptor [PR] negative, HER2 negative, Ki-67 40\%; left: invasive ductal carcinoma with DCIS, ER/PR positive, HER2 negative, Ki-67 10\%) was referred for cardiology consultation after surgical ablation of both sides for cardiac risk evaluation for chemotherapy.

Pre-existent hypertrophic cardiomyopathy with mesoventricular obstruction and restrictive diastolic function was known, with a left ventricular outflow tract gradient of $45 \mathrm{~mm} \mathrm{Hg}$ accompanied by moderate to severe eccentric mitral regurgitation due to dynamic systolic anterior mitral leaflet motion (SAM) caused by the turbulent flow (Fig. 1). A history of stable angina pectoris on exertion, chronic slightly elevated troponin and previous cardiac decompensation with leg edema was present, as well as frequent ventricular extrasystoles without syncope and paroxysmal atrial fibrillation. A previous coronary angiography had excluded severe coronary stenosis. The ECG was remarkable with T-wave inversions in the chest leads.

With intensified cardiac monitoring in addition to the usual oncology follow-up, with echocardiography and serum biomarkers troponin as well as NTproBNP before each cycle, the patient was able to undergo 4 cycles of epirubicin $\left(4 \times 90 \mathrm{mg} / \mathrm{m}^{2}\right)$ and cyclophosphamide $\left(4 \times 600 \mathrm{mg} / \mathrm{m}^{2}\right)$, followed by paclitaxel adjuvant (2 weeks per $80 \mathrm{mg} / \mathrm{m}^{2}$ ) and docetaxel $\left(4 \times 100 \mathrm{mg} / \mathrm{m}^{2}\right)$. After the 3rd cycle of anthracycline, the patient reported intermittent dyspnea; however, no significant change in left ventricular function or biomarkers was observed and peripheral edema was not present. Due to low blood pressure, the cardiac medication was reduced intermittently. Therefore, the 4th cycle could be completed. Radiation is currently ongoing.

The current case shows that even high-risk cardiac patients can undergo their life-saving oncology treatment with close observation and monitoring. 

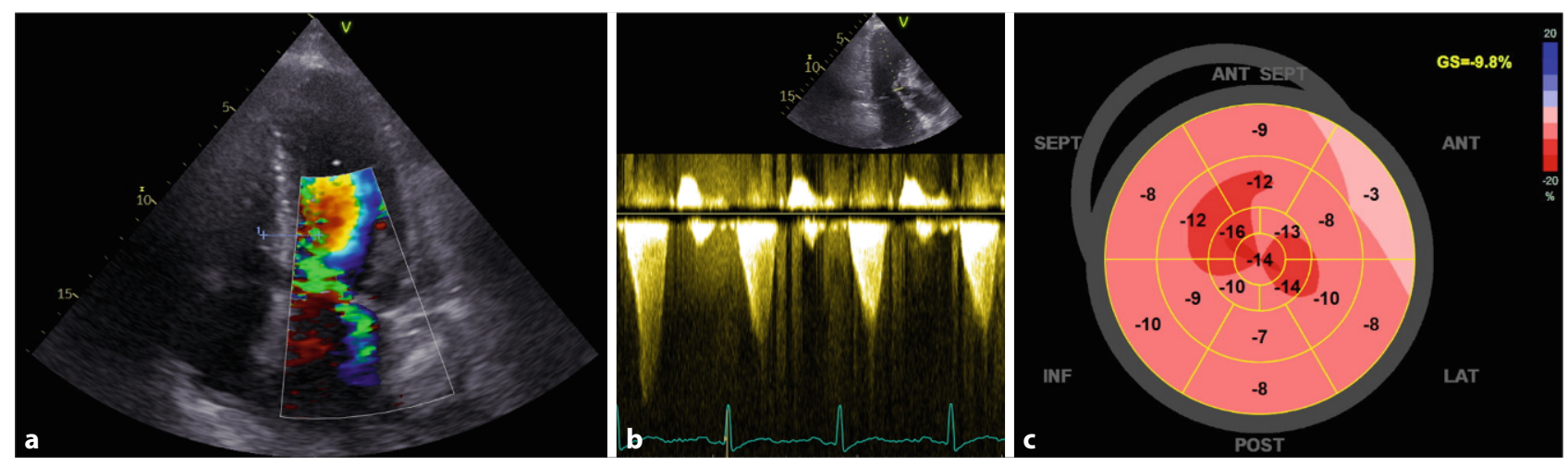

Fig. 1 Patient with hypertrophic obstructive cardiomyopathy undergoing chemotherapy for bilateral high-risk breast cancer (a echocardiography showing excentric mitral regurgitation

\section{Conclusion}

Early risk assessment and cardiac medication optimization without delaying the vital begin of oncology treatment can help to prevent cardiotoxicity development. While the frequency depends on the patient's baseline risk, assessment at least of the simple serum biomarkers NT-pro/BNP and troponin, as well as evaluation of left ventricular function and strain if available by echocardiography are helpful in predicting and detecting cardiac deterioration [12]. Increased cardiac therapy and oncology therapy adaptation if possible may enable continuation of treatment cycles [13]. More resources are needed for the establishment of dedicated cardio-oncology units.

\section{Take home message}

Baseline cardiac risk assessment in all and close follow up NT-proBNP, troponin, and echocardiography is recommended in high risk patients with cardiotoxic therapies. In survivors long-term cardiac follow up is necessary.

Funding Open access funding provided by Medical University of Vienna.

Conflict of interest J. Bergler-Klein declares that she has no competing interests.

Open Access This article is licensed under a Creative Commons Attribution 4.0 International License, which permits use, sharing, adaptation, distribution and reproduction in any medium or format, as long as you give appropriate credit to the original author(s) and the source, provide a link to the Creative Commons licence, and indicate if changes were made. The images or other third party material in this article are included in the article's Creative Commons licence, unless indicated otherwise in a credit line to the material. If material is not included in the article's Creative Commons licence and your intended use is not permitted by statutory regulation or exceeds the permitted use, you will need to obtain permission directly from the copyright holder. To view a copy of this licence, visit http://creativecommons.org/licenses/by/4.0/. with turbulent flow in the ventricular outflow tract; $\mathbf{b}$ continuous wave Doppler obstructive gradient; c example of global strain bulls-eye)

\section{References}

1. Anker MS, Hadzibegovic S, Lena A, et al. Recent advances in cardio-oncology: a report from the 'Heart Failure Association 2019 and World Congress on Acute Heart Failure 2019'. ESC Heart Fail. 2019;6(6):1140-8.

2. Frey M, Bergler-Klein. Echocardiographic evaluation of patients undergoing cancer therapy. Eur Heart J Cardiovasc Imaging. 2021;22(4):375-82.

3. Lyon AR, Dent S, Stanway S, et al. Baseline cardiovascular risk assessment in cancer patients scheduled to receive cardiotoxic cancer therapies: a position statement and new risk assessment tools from the Cardio-Oncology Study Group of the Heart Failure Association of the European Society. Eur J Heart Fail. 2020;22(11):1945-60.

4. Čelutkienè J, Pudil R, López-Fernández T, et al. The role of cardiovascular imaging in cancer patients receiving cardiotoxic therapies: a position statement on behalf of the Heart Failure Association (HFA), the European Association of Cardiovascular Imaging (EACVI) and the Cardio-Oncology Council of the European Society of Cardiology (ESC). Eur J Heart Fail. 2020;22(9):1504-1524. https:// doi.org/10. 1002/ejhf.1957.

5. Okwuosa TM, Keramida K, Filippatos G, Yancy CW. Cancer therapy and the heart; the necessity to calibrate risk. Eur J HeartFail. 2020;22(11):1961-5.

6. PudilR, MuellerC, ČelutkienèJ, etal. Roleofserumbiomarkers in cancer patients receiving cardiotoxic cancer therapies: a position statement from the Cardio-Oncology Study Group of the Heart Failure Association and the CardioOncology Council of the European Society of Cardiology. EurJ HeartFail. 2020;22(11):1966-83.

7. Bergler-Klein J. Real-life insight into ibrutinib cardiovascular events: defining the loose ends. J Am Coll Cardiol. 2019;74(13):1679-81.

8. De Boer RA, Hulot JS, Tocchetti CG, et al. Common mechanistic pathways in cancer and heart failure. A scientific roadmap on behalf of the Translational Research Committee of the Heart Failure Association (HFA) of the European Society of Cardiology (ESC). Eur J Heart Fail. 2020;22(12):2272-89.

9. Meijers WC, Maglione M, Bakker SJL, et al. Heart failure stimulates tumor growth by circulating factors. Circulation. 2018;138(7):678-91.

10. Hasin T, Gerber Y, Weston SA, et al. Heart failure after myocardial infarction is associated with increased risk of cancer. JAm Coll Cardiol. 2016;68(3):265-71. 
11. Aboumsallem JP, Moslehi J, de Boer RA. Reverse cardio-oncology: cancer developmentin patients with cardiovascular disease. JAm HeartAssoc. 2020;9(2):e13754.

12. Bergler-Klein J. Strain as hallmark to prevent interruption of breast cancer therapy. Eur Heart J Cardiovasc Imaging. 2019;20(12):1353-4.

13. Cardinale D, Colombo A, Bacchiani G, Tedeschi I, Meroni CA, Veglia F, et al. Early detection of anthracycline cardiotoxicity and improvement with heart failure therapy. Circulation. 2015;131(22):1981-8.

Publisher's Note Springer Nature remains neutral with regard to jurisdictional claims in published maps and institutional affiliations. 